\title{
Entrelacs
}

Cinéma et audiovisuel

10 | 2013

Le Toucher

\section{Entre la « main de l'œil » et l' « œil digital », proximité et profondeur : la dimension haptique à l'horizon du Cinéma 3D et des acteurs virtuels}

Isabelle Rieusset-Lemarié

\section{OpenEdition}

Journals

Édition électronique

URL : http://journals.openedition.org/entrelacs/538

DOI : 10.4000/entrelacs.538

ISSN : 2261-5482

Éditeur

Éditions Téraèdre

Référence électronique

Isabelle Rieusset-Lemarié, «Entre la « main de l'œil » et l' « œil digital », proximité et profondeur : la dimension haptique à l'horizon du Cinéma 3D et des acteurs virtuels », Entrelacs [En ligne], 10 | 2013, mis en ligne le 12 septembre 2013, consulté le 19 avril 2019. URL : http://journals.openedition.org/ entrelacs/538; DOI : 10.4000/entrelacs.538

Ce document a été généré automatiquement le 19 avril 2019.

Tous droits réservés 


\title{
Entre la « main de l'œil » et l' « œil
} digital ", proximité et profondeur : la dimension haptique à l'horizon du Cinéma 3D et des acteurs virtuels

\author{
Isabelle Rieusset-Lemarié
}

\section{Proximité et/ou profondeur: l'ambivalence du Cinéma 3D au regard de l'haptique deleuzien}

\subsection{Proximité ou profondeur : les « deux écoles » du Cinéma 3D}

L'effet de relief du Cinéma 3D renforce la sensation d'un « élément tactile dans l'optique elle-même ${ }^{1}$ » en laquelle Walter Benjamin voyait une des caractéristiques du cinéma. On retrouve cette « réunion des deux sens, le toucher et la vue ${ }^{2}$ » caractéristique de ce que Deleuze appelle « la fonction tactile, ou plutôt haptique ${ }^{3}$ » qui « permet à l'œil de procéder comme le toucher ${ }^{4}$ ", ce que Valéry appelle la «main de l'œil ${ }^{5}$ ». Cette sensation du rugueux offerte à la «main de l'œil» est particulièrement présente dans le film de Werner Herzog (La grotte des rêves perdus, 2010) qui met en œuvre l'effet 3D au cinéma pour exacerber cette impression de toucher les anfractuosités de la roche. Ce film ressortit, à bien des égards, à l'haptique deleuzien qu'il vérifie, en particulier, dans cette façon de nous confronter à une forme de proximité. Pour Deleuze, en effet, l'haptique se caractérise par un «espace de proximité [...] comme l'expression d'une "vision rapprochée" [...] c'est un espace sans profondeur [...] qui permet au regard de palper l'objet » [et qui s'oppose à] une "vision éloignée", se déployant dans un espace optique ou strié. ${ }^{\circ}$ " De nombreux éléments de La grotte des rêves perdus ${ }^{7}$ montrent non seulement qu'on peut considérer ce film comme vérifiant les propriétés de l'haptique deleuzien mais qu'il s'agit en outre de «reconnaître dans le documentaire de Werner Herzog ce qui le 
distingue, autrement dit sa "force haptique" et transgressive telle qu'elle est intensifiée par l'usage de la technologie 3-D ${ }^{8}$ ».

Pour autant, on ne saurait caractériser l'ensemble des réalisations du Cinéma 3D par cet haptique deleuzien. Dans la « nouvelle 3D ${ }^{9}$ » au cinéma il y aurait, manifestement, deux " écoles ", entraînant des divergences de sensibilité chez leurs amateurs respectifs. La pluralité du Cinéma 3D tend à exacerber cette proximité et cette ambiance fusionnelle (caractéristiques de l'haptique deleuzien), mais elle tend également à exacerber l'impression de profondeur ressortissant à l'espace optique. Les polémiques suscitées par la sortie de Titanic en 3D ont conduit certains bloggeurs à défendre la profondeur comme la spécificité de la 3D :

[...] si aucun "jumper" ne vous a sauté au visage, c'est peut-être justement que l'intérêt de la 3D n'est pas que l'image vous saute aux yeux, mais bien que vous puissiez rentrer dedans. L'unique plus-value de la 3D réside à mon sens dans la profondeur de champ, bien plus que dans l'esbroufe de quelques éléments que vous pourriez presque "toucher". ${ }^{10}$

Partisan de la même approche, Caroline Champetier, pour qui «la 3D, ce n'est pas ce qui [...] vous tombe dessus quand vous regardez le film, c'est au contraire quelque chose qui doit offrir une profondeur ${ }^{11}$ ", valorise le gouffre d'Avatar " qui est derrière le point de convergence et s'éloigne de nous ", lorsque d'autres préfèrent Werner Herzog parce qu'il " projette ce gouffre au devant de nous comme s'il allait nous tomber dessus afin de susciter le désir transgressif de toucher ce qui se présente à nous. [...] Le gouffre au fond se présente devant. C'est parce que l'image est, à l'instar de l'aura selon W. Benjamin, ce lointain aussi proche fût-il ${ }^{12}$ ».

\subsection{Les effets paradoxaux du nouveau cinéma 3D}

4 Le cinéma 3D inaugure-t-il une " profondeur haptique " produisant des effets paradoxaux où le sentiment de proximité tactile ne crée pas du «sans distance » car il rapproche le lointain « en tant que lointain ${ }^{13}$ " ? Certaines œuvres du Cinéma 3D contemporain tendent à montrer qu'on peut explorer la profondeur tout en valorisant les perceptions haptiques. Non seulement on pourrait conjuguer textures en relief des premiers plans et effets de profondeur ${ }^{14}$, mais la «nouvelle 3D » serait capable de faire surgir des effets haptiques dont le dynamisme est d'autant plus fort qu'il surgit des profondeurs pour faire irruption, au-delà de l'écran, dans l'espace de la salle, à proximité immédiate du spectateur. Telle est la nouvelle potentialité de ce que nous qualifions de "profondeur haptique", irréductible à l'opposition dualiste entre profondeur de l'espace (censée être caractéristique de l'espace optique perspectif) et vision rapprochée du «regard haptique ».

Dans Mille plateaux, cette vision rapprochée, caractéristique de l'haptique selon Deleuze et Guattari, fonderait un espace sans contour ni limite : « Là où la vision est proche, l'espace n'est pas visuel, ou plutôt l'œil lui-même a une fonction haptique et non optique : aucune ligne ne sépare la terre et le ciel, qui sont de même substance; il n'y a pas d'horizon, ni de fond, ni perspective, ni limite, ni contour ou form $\mathrm{e}^{15} »$. On retrouve dans certaines scènes du film en 3D de Ang Lee, L'odyssée de Pi (2012), les caractéristiques de l'espace haptique telles qu'elles sont décrites dans ce passage de Mille plateaux (le ciel et la mer se confondent, les contours s'estompent, il n'y a pas de point de fuite à l'horizon) à une exception près, majeure. En effet, ces éléments haptiques que Mille plateaux décrit comme 
ne pouvant émerger que d'une "vision proche » n'en apparaissent pas moins liés, dans ces images de l'Odyssée de $P i$, à une " vision éloignée » qui fait ressentir au spectateur tant la distance qui le sépare du héros debout sur sa barque, que l'éloignement de celui-ci de toute terre, perdu dans l'immensité cosmique de ce milieu ambiant où ciel et mer sont indiscernables. Si ces scènes ressortissent au nouveau mode de la «profondeur haptique ", il n'y a pas, pour autant, de perception de profondeur, au sens relatif à la perspective, dans la mesure où il n'y a pas de ligne d'horizon et que le personnage semble comme «perdu » dans une substance lumineuse sans repères. Ce type d'images dans ce film ${ }^{16}$ déconstruit l'association traditionnelle entre "vision éloignée " et profondeur perspectiviste. Il échappe en outre à l'opposition entre ce que l'on croyait déjà être les « deux écoles » du Cinéma 3D, celle de la profondeur et celle de la vision rapprochée vous « tombant dessus ». Cet usage inédit a montré que l'esthétique de la 3D au cinéma n'en est encore qu'à ses balbutiements et que certaines œuvres contribuent à enrichir son vocabulaire, comme l'a noté avec pertinence Jean-Michel Frodon. ${ }^{17}$

Si les pistes d'analyses de Mille plateaux s'avèrent heuristiques en rendant intelligibles les troubles perceptifs engendrés par ce type de mise en œuvre haptique de l'image, leur cadre d'interprétation dualiste ne leur permet pas d'anticiper ce découplage entre « espace haptique » et "vision rapprochée » mis en œuvre par la «nouvelle 3D » au cinéma.

\section{En deçà de l'haptique deleuzien : un modèle dualiste à dépasser?}

\subsection{Les écueils de l'assimilation de la fonction haptique à un " espace lisse "}

7 Si les notions d'« espace optique » et d'« espace haptique » apparaissent heuristiques, leur assimilation respective à un « espace strié » et un « espace lisse $\|^{18}$ ne manque pas de faire problème. Dans Mille Plateaux, Deleuze et Guattari disent emprunter cette opposition entre «espace lisse » et «espace strié » à Pierre Boulez ${ }^{19}$. Si, dans ce contexte musical précis, ce couple notionnel peut avoir une pertinence particulière, son extension systématique dans Mille plateaux, non seulement à un "modèle musical», mais aux différents modèles technologique, maritime, mathématique, physique et esthétique, laisse transparaitre le « coup de force » rhétorique de cette généralisation dont la rigueur épistémologique n'est plus probante. La pertinence analytique s'appauvrit à mesure que s'y renforce une dichotomie axiologique qui tend à figer ces oppositions duelles en un système, sinon manichéen ${ }^{20}$, à tout le moins dualiste entre deux forces qui s'affrontent ${ }^{21}$. Si cette mise en scène agonistique, implicitement politique, peut s'avérer pertinente lorsque se décrit la tendance à la disparition des cultures nomades (assimilées par Deleuze et Guattari au "lisse») sous la pression dominante des cultures sédentaires (assimilées par Deleuze et Guattari au "strié »), elle s'avère souvent "en porte à faux » dès lors qu'il s'agit d'appréhender les enjeux, esthétiques, de l'haptique. L'inadéquation de ce modèle global à la caractérisation de l' "espace haptique" se trahit par l'assimilation à laquelle elle conduit entre le "feutre» et le "patchwork» sous la catégorie du « lisse », pour caractériser l'haptique. L'exemple du patchwork « qui joue de la texture des tissus ${ }^{22}$ » et des « quilts à pièces rapportées ${ }^{23}$ » semble congruent avec une sollicitation privilégiée de la fonction haptique. Mais comment admettre de qualifier le 
relief de cette multiplicité géométrique (aux «effets de 3D» récurrents, dans une esthétique fragmentée) comme un "espace lisse»! Appliqué à l'espace haptique, ce terme d'« espace lisse » apparaît, manifestement, mal choisi.

Significativement, l'expression d' « espace lisse » n'apparait pas dans l'essai de Deleuze ( Francis Bacon/Logique de la sensation). On doit distinguer, de ce point de vue, l'incidence de ce livre de celle de l'ouvrage co-rédigé par Deleuze et Guattari (Mille plateaux). Alors que ce dernier tend, pour l'essentiel, à ériger ce modèle d'oppositions duelles en un " système ", au risque de le figer en une axiologie dualiste, le livre sur Francis Bacon reste fondamentalement ancré sur l'analyse d'œuvres singulières de peintres et tend à présenter les généralisations avancées plus comme des hypothèses, des pistes d'interprétations, que comme les vérités assénées d'un système axiologique figé. Si les modalisations présentes dans Mille plateaux ${ }^{24}$ restent recouvertes par la prégnance du système duel d'opposition, celles du livre de Deleuze sur Francis Bacon ne cessent d'inviter à une distance critique évitant d'appréhender ces oppositions de façon manichéenne :

On aurait tort toutefois d'opposer les deux tendances, vers un espace optique pur, vers un espace manuel pur, comme si c'était des incompatibles. [...] il suffit de regarder à l'envers et de près un Rembrandt pour découvrir la ligne manuelle comme l'envers de la lumière optique. On dirait que l'espace optique a lui-même libéré de nouvelles valeurs tactiles (et inversement aussi). ${ }^{25}$

Cette valorisation des formes hybrides, très affirmée dans ce livre, produit des échos dans le cadre de Mille plateaux lorsqu'est souligné « comment l'espace échappe aux limites de son striage ${ }^{26}$ ». L'exploration de cette déconstruction des espaces striés, redonnant sa puissance à la fonction haptique, s'est particulièrement développée dans le champ de création contemporaine des courts métrages. Dans Inanis Aedes ${ }^{27}$, non seulement ces éléments associés à l'« espace lisse » (vent, fluides, roche, lumière, etc.) sont déterminants mais d'autres scènes ne s'attachent à installer le cadre d'un espace strié (marqué par les verticales et les horizontales d'une architecture rationnelle) que pour mieux le déconstruire, en jouant de ses interstices d'où émergent deux flux torrentiels d'une eau tumultueuse, qu'on pense un instant voir se rejoindre pour former une nouvelle verticale, mais dont l'éclaboussement haptique - rendu particulièrement sensible par ce court métrage en 3D - finit par déborder ce cadre quadrillé et par substituer à cet agencement de lignes une dynamique explosive irréductible à ce striage. Un autre court métrage joue $\mathrm{du}$ « splitscreen » comme d'une « ligne de déterritorialisation ${ }^{28}$ » qui passe « entre » deux territoires et où la migration de l'un à l'autre " jouit d'une puissance de métamorphose ${ }^{29}$ ». Ces courts métrages manifestent la tendance à transgresser l'opposition duelle entre « espace strié » et « espace lisse ».

Significativement, la dernière phrase de l'ouvrage de Deleuze sur Francis Bacon insiste sur le dépassement de la dualité de l'optique et du tactile que cristallise la fonction haptique $^{30}$. Dans L'image-temps Deleuze ira plus loin encore dans cette mise en garde contre une opposition axiologique manichéenne tendant à stigmatiser l'œil au profit du tactile en rappelant l'ambivalence de ce dernier : « c'est le tactile qui peut constituer une image sensorielle pure, à condition que la main renonce à ses fonctions préhensives et motrices pour se contenter d'un pur toucher ${ }^{31} »$. 


\subsection{La rationalisation de l'œil digital et la main démiurgique}

11 Face aux « images-projectiles en 3D », de jeunes enfants ${ }^{32}$ tendent les mains pour saisir ce qu'ils ressentent comme des objets plus que comme des images. L'acclimatation des jeunes générations au tactile dans les nouveaux médias tend à privilégier une posture de « saisie » par la main active, plutôt qu'une appréhension par la « main de l'œil » face à ces « images-objets» qui sortent de l'écran. C'est en vue des habitus sensoriels de ces générations qu'a été conçu un «écran qui permettrait de "toucher" les images 3D qu'il diffuse $»^{33}$. En quoi ces images destinées à la main s'adressent-elles à un « œil digital»? Ce type de nouveau média ne ferait que confirmer la tendance selon laquelle «l'œil du sujet spatialisateur est greffé sur sa $\operatorname{main}^{34}$ ». Dans ces nouveaux médias numériques tactiles, la fonction d'organisation rationnelle de l'espace qui était dévolue au regard, selon le code de la perspective, tend à être prise en charge non pas tant, par la main, que par ses doigts. Dans ce nouveau paradigme l' " œil digital ", greffé au bout des mains, opère une fonction de discrimination des signes discrets. Ces nouveaux médias tactiles ne s'adressent pas tant à la sensorialité du toucher diffus de la main qu'à l'expérience des doigts habitués aux claviers digitaux qui les transforment en agents d'écriture subordonnant le tactile à une organisation sémiotique de l'espace. Dans ce contexte, le modèle du digital est celui, sémiotique, $d u \operatorname{code}^{35}$. Il manifeste la nouvelle efficace de cet ordre rationnel digital. Exemplaire de cette tendance, le «miroir tactile ${ }^{36}$ » transforme cette surface réflexive, où se mire la construction du sujet, en un espace d'incrustation numérique des informations de tous types s'ordonnant rationnellement à la commande du doigt qui les fait apparaître. Dans ce contexte, le doigt devient le meilleur agent du quadrillage de l'espace optique et du « Strié », au sens deleuzien. L'« œil digital » interagit avec des écrans tactiles pré-codés qui s'activent sous sa pression et répondent à sa commande. Les signes activables de ces écrans tactiles obéissent non plus au doigt et à l'œil mais à cette nouvelle instance hybride : l' « œil digital ».

L'exacerbation du tactile, dans l'idéologie de la "première interactivité ${ }^{37}$ ", s'est développée comme une volonté de puissance manipulatoire. En filigrane de l' «œil digital ", ce qui est convoqué c'est la «main démiurgique». On flatte l'usager de ces nouvelles interfaces tactiles en conférant à l'interacteur l'illusion de cette puissance démiurgique qui était, traditionnellement, réservée à l'auteur ${ }^{38}$. Alors que dans le cinéma des premiers temps, Cohl pouvait jouer de l'exhibition de sa main démiurgique, capable de donner vie par son dessin au monde inanimé, ces médias numériques tactiles donnent l'illusion à leurs usagers d'avoir troqué leur position passive de spectateurs pour celle d'un auteur démiurgique peuplant cet espace de formes animées à leur commande, lors même que celle-ci n'obéit qu'à la pré-programmation d'un espace visuel dont ils se contentent d'activer les fonctionnalités par une posture qui se réduit à une «interactivité de presse boutons », ces derniers fussent-ils « cachés » et n'apparaissant qu'au gré de leur gestualité intuitive.

13 Signe visuel, créature virtuelle ou simple source d'information, quel qu'il soit, l'objet «à portée de main interactive » serait ce qui répond "à la commande » et qui induit, en retour, une illusion de maîtrise. Littéralement, en allemand, la Zuhandenheit c'est ce qui est «à portée de main », ce qui rentre dans le champ d'utilisation de la main, ce qui est disponible comme outil manipulable, préhensible ${ }^{39}$. Si Deleuze articule la fonction haptique sur la proximité, ce n'est pas sans être conscient des ambivalences de ce qu'elle 
peut produire dès lors qu'elle s'en tient à l'horizon du manipulable ou du préhensible, à cette idéologie utilitariste du «à portée de main ${ }^{40}$.

Pour autant, le toucher ne se réduit pas à cet empire de commande de la main qui n'aurait de cesse que de mettre en contact, de rapprocher ce qui était hors de prise pour le soumettre à la commande. Cela passe par une certaine appréhension du geste, non pas du geste prédateur, mais du geste porrection ${ }^{41}$, du geste gestation, du geste qui prend soin et qui porte à terme ${ }^{42}$. Mais cela passe, aussi, par une réexploration du toucher, en tant qu'il ne se réduit pas à l'identification à la saisie, à la prise, à la captation manipulatoire.

\section{Empathie et acteurs virtuels : les nouvelles modalités de la profondeur haptique}

\subsection{L'empathie kinesthésique singulière de la « performance capture »}

Sans retomber dans une axiologie binariste, on peut cependant noter que le degré de mise en œuvre du toucher est plus fort dès lors qu'il ne se contente pas d'obtenir une action sur l'image par la pression d'un doigt et qu'il engage l'expérience disséminée dans le corps tout entier que constitue la kinesthésie et sa possible ouverture à une empathie kinesthésique ${ }^{43}$. James Cameron a réussi à créer cet effet d'empathie en contournant la "fausse proximité » réaliste, et son risque à provoquer l' "uncanny valley ${ }^{44}$ ", mais également en ouvrant la voie à l'« e-motion capture ${ }^{45} »$. On doit souligner dans Avatar (2009) la "profondeur haptique» des acteurs virtuels, en ce sens que leur corps laisse transparaître les gestes des acteurs réels - et la kinesthésie interne dans laquelle ils sont ancrés - qui ont permis leur gestation grâce à un perfectionnement de la "performance capture $^{46}$ ». On peut se demander dans quelle mesure la sensation de ce "toucher interne " n'est pas encore plus accessible avec ces acteurs virtuels d'Avatar qu'avec des acteurs réels. Il est cependant difficile de savoir exactement à quoi cela tient. On peut faire plusieurs hypothèses.

L'horizon d'attente, pour un acteur virtuel, n'est pas le même. Il suffit que l'acteur virtuel donne la sensation interne de son corps lorsqu'il marche pour qu'on ressente une surprise, une émotion, alors qu'il ne suffira pas à un acteur réel d'être mis dans cette situation, pour lui, ordinaire, de la marche, et qu'il lui sera plus facile de nous communiquer cette empathie kinesthésique s'il danse, de façon plus ou moins lascive, ou s'il est diégétiquement surdéterminé par une relation sensorielle intersubjective.

La seconde raison tient à l'effet de « transparence » du corps des acteurs virtuels. Le corps d'un acteur réel reste « opaque " au sens où le spectateur n'est pas mis en situation de focaliser son imagination sur l'intérieur organique de ce corps. Le ferait-il que cette représentation mentale de la "mécanique " biologique jouerait plutôt l'effet d'une distanciation, faisant obstacle à l'image d'un ressenti propre à la façon dont un sujet vit son corps de façon émotionnelle et sensorielle et, non pas, organique ${ }^{47}$. En revanche, la publicité faite sur la méthode de la "performance capture » dont sont issus les acteurs virtuels d'Avatar conduit à voir se profiler, sous leur peau, la subjectivité interne des acteurs réels qui animent leurs gestes. L'intérieur du corps virtuel de ces acteurs nous renvoie, non pas à une machinerie organique, mais à une puissance d'animation du geste ressortissant à la modalité kinesthésique du toucher. Sous leur peau opère la pure 
expression du toucher, les signes intenses issus de la capture de ces expressions gestuelles et tactiles. Leur « corps interne » est lui-même « pur geste ».

La troisième raison tient à la densité du regard des créatures virtuelles d'Avatar ${ }^{48}$, lors même que l'on croyait, antérieurement à ce film, que cette intensité était réservée aux acteurs réels. Or, percevoir, non pas, seulement, des yeux, mais un regard, ne relève pas seulement d'une impression optique. Cela demande de sentir une profondeur. Celle-ci peut s'interpréter en termes d'intentionnalité prêtée à cet "alter ego". Mais la profondeur que l'on prête à un autre sujet ne saurait se suffire d'un schème dualiste et ne lui prêter qu'un « esprit ». Cette conscience ne peut s'exprimer dans son regard que parce que ce sujet est incarné dans un corps. Dès lors, en filigrane de cette profondeur de l'intentionnalité, se profile aussi une "profondeur haptique ", ancrée sur la kinesthésie que met en jeu la façon dont ce sujet s'éprouve comme tel, en tant qu'il se "sent" exister de l'intérieur. À ce titre, le geste de quelqu'un, en tant qu'expression d'une intentionnalité qui s'adresse à vous, est aussi une forme de regard. Le geste, c'est le regard du toucher. Le regard de l'autre, qu'il soit issu de son œil ou de ses gestes, est porteur de son intériorité. Percevoir le regard de quelqu'un comme tel, c'est être sensible à son intériorité, tant «mentale » que « kinesthésique », dans l'articulation indissociable de ces deux modalités.

\subsection{Le « regard haptique » comme exploration empathique du vertige de l'altérité}

Par rapport à cette " réussite » du regard des créatures virtuelles d'Avatar, l' « absence de regard » du tigre de synthèse de L'Odyssée de Pi pourrait se présenter comme un « échec ». Le réalisateur a montré qu'il était conscient qu'on ne peut pas " tricher ${ }^{49}$ » avec le regard d'un tigre. Cependant, l'habileté d'Ang Lee a consisté à transformer ce "défaut » en un parti pris intéressant qui nous permet d'approcher la question de l'empathie "par défaut ». En effet, un des moments clés de l'histoire de ce film réside dans le dessillement de la croyance du héros par son père. Alors que le jeune Pi a l'illusion de communiquer de regard à regard, comme " d'homme à homme " avec les tigres, son père tente de lui faire prendre conscience qu'il projette un leurre anthropomorphique sur cet animal et ne fait que lui « prêter » un regard. Dès lors l'effet « d'absence de regard » du tigre de synthèse, pendant la cohabitation avec cet animal sur la barque, semble confirmer la justesse de l'avis paternel. Le jeune héros vit une sorte d'initiation à la reconnaissance de l'altérité radicale de l'animal. Alors qu'il a tenté de tisser des relations " humaines » avec ce tigre et qu'il s'y est " attaché », ce dernier part sans se retourner, " sans un regard », dès qu'il a la possibilité de s'échapper dans un milieu naturel qui lui est propice. L'empathie absolue ne serait pas possible entre humain et tigre; a fortiori la sympathie prétendant vous donner l'illusion de connaître le perçu interne de cet animal. Loin de jouer de la facilité d'une empathie "à la Disney ", faite de projections anthropomorphiques sur des figures animales, Ang Lee nous confronte à l'altérité radicale de l'animal, redoublée par l'altérité sans commune mesure d'une créature de synthèse. Face à cette altérité radicale, l'empathie, à tout le moins dès lors qu'on la réduit à une posture d'identification, ne serait qu'un leurre. Mais, par cette approche «par défaut» de l'empathie identificatoire, Ang Lee ne nous ouvre-t-il pas à l'exigence d'une empathie plus radicale qui puisse postuler un "se mettre à la place de l'autre » tout en respectant sa radicale différence «en tant qu'autre». Le chemin initiatique de l'empathie radicale ne serait pas tant de 
s'identifier à des « figures du double » que de faire l'expérience de l'altérité radicale. De ce point de vue, le film d'Ang Lee nous confronte à une des modalités les plus exigeantes de l'empathie, par le biais de l' « absence de regard » de ce «tigre de synthèse ».

Cette confrontation empathique qui maintient la distance radicale de l'autre « en tant qu'autre » recroise la capacité à rapprocher le lointain « en tant que lointain ». Alors que les nouveaux supports tactiles jouent de la proximité comme d'une forme d'instrumentalisation digitale, le Cinéma 3D, en tant qu'il assume son effet de distanciation $^{50}$, apprivoise la distance radicale sans jamais la domestiquer. Si le Cinéma, fût-il d'animation, fût-il en 3D, ne peut rivaliser avec le plaisir manipulatoire offert par les tablettes tactiles, il reste en position privilégiée d'explorer la profondeur du désir. Plus on semble se rapprocher de l'autre, plus on semble s'ouvrir à une empathie, parfois même kinesthésique, plus on perçoit la distance vertigineuse avec cette altérité radicale. Mais c'est de ce vertige même que renaît sans cesse le désir. Le "regard haptique " propre au cinéma ne serait plus tant, dans ce cas, l'effacement de la profondeur que son exploration vertigineuse, sous le signe du désir.

21 La «main de l'œil » permet de percevoir le caractère vertigineux de cette "profondeur haptique » où se profile une altérité radicale. Encore faut-il qu'elle soit confrontée non pas, seulement, à des objets inertes offerts à une manipulation instrumentaliste, mais à des créatures dotées, elles mêmes, d'une capacité sensorielle tactile. Seul le «toucher réciproque », fût-il imaginé, permet la résonance ${ }^{51}$ émotionnelle de l'empathie. De là l'importance de confronter le spectateur ou l'interacteur non plus seulement à des « objets virtuels » mais à des «personnages virtuels » capables, de par l'autonomie et le " gestus $^{52}$ » qui leur ont été conférés, d'adopter une véritable posture. Dans l'acception deleuzienne du "gestus ${ }^{53}$ ", l'exigence à ne pas dépendre de la détermination d'un scénario préalable imposant ses faits et gestes à des "pantins " manipulés concerne les acteurs et les personnages dont l'interaction doit rester libre et pouvoir secréter une histoire. Pour Deleuze, le "gestus» est indissociable de l' « exigence d'un cinéma des corps ». Celle-ci s'avère propice à une empathie tactile.

En revanche, dès lors qu'elles sont soumises à une manipulation démiurgique, que ce soit par le scénario ou par une interactivité "presse bouton", les créatures virtuelles ne peuvent nous donner qu'un leurre de réactivité tactile incapable de produire cette sensation d'un "toucher réciproque" s'adressant à un autre doté d'une autonomie sensorielle et d'une capacité à générer des gestes. Si différents modes d'approches, techniques ou narratifs, sont possibles, l'essentiel est de doter ces créatures virtuelles d'une "intériorité tactile», d'une "profondeur haptique ». Si celle-ci n'est pas obligée d'être à l'image de l'intériorité humaine ${ }^{54}$, elle doit permettre de confronter le spectateur non plus à des objets inertes mais à une créature dont l'intelligence perceptive est tangible. En somme, pour que les créatures virtuelles sollicitent un "regard haptique », il ne suffit pas qu'elles soient mises en scène dans un "espace haptique», il faut qu'elles soient dotées d'une "profondeur haptique ». Telle est la voie ouverte tant aux courts et longs métrages en 3D qu'aux nouveaux environnements tactiles, dès lors qu'il s'agit de dépasser la rationalisation de l' « œil digital " pour redonner sa pleine dimension à la potentialité empathique du toucher. 


\section{NOTES}

1. W. Benjamin, «L'œuvre d'art à l'époque de sa reproduction mécanisée (1936) ", in Ecrits français, Gallimard, 1991, p. 183.

2. G. Deleuze, Francis Bacon/Logique de la sensation, Editions du Seuil, 2002, p. 115.

3. Ibid., p. 115. (Rappelons que haptique vient du verbe grec aptô qui signifie toucher.)

4. Ibid., p. 115.

5. H. Parret, «Spatialiser haptiquement : de Deleuze à Riegl, et de Riegl à Herder ", in Nouveaux Actes Sémiotiques, Prépublications 2008-2009 : Sémiotique de l'espace : «Voici ce que Valéry écrit dans les Cahiers (II, 1301) [...] : « De ces formes sur quoi la main de l'œil passe et qu'elle éprouve, selon le rugueux, le poli, le nu, le poilu, le coupant, le mouillé et le sec ». Il ne s'agit même pas de la main dans l'œil mais de la main de l'œil, de l'œil qui devient, sans cesser d'être un œil, une main experte ».

6. M. Buydens, «Espace lisse/Espace strié », in Le vocabulaire de Gilles Deleuze (R. Sasso et A. Villani, dir.), Les Cahiers de Noesis, $n^{\circ} 3$, Printemps 2003, pp. 130-134.

7. Franz B., « Des nouvelles du front cinématographique (59) : La caverne aux images, deux films sur et dans la grotte Chauvet», 12 septembre 2011: «En même temps, son usage de la technologie 3-D restitue, par delà la simple visibilité des peintures pariétales, un tact qui en actualise encore plus intensément la puissance esthétique. Cette tacticité est une "tactilité" qui est le propre du geste de cinéma pratiqué par Werner Herzog, comme l'a montré Gilles Deleuze à la suite d'Emmanuel Carrère (cf. Werner Herzog, éd. Edilig, 1982, p. 25) : [...] «Chez Herzog, on assiste à un effort extraordinaire pour présenter à la vue des images proprement tactiles. » (in Cinéma 2. L'image-temps, Ed. de Minuit, coll. «Critique », 1985, p. 22). Ce que le philosophe appelle ailleurs haptique [...], Werner Herzog le trouve dans la grotte Chauvet, dont les peintures témoignent de l'utilisation esthétique des anfractuosités de la roche [...] Et cette dynamisation est très précisément prolongée par la 3-D qui en autorise ainsi le partage avec le spectateur. »

8. Ibid.

9. Rappelons que l'usage de la 3D remonte au cinéma des premiers temps ( $c f$. non seulement le « remake » en anaglyphe de leur film L'arrivée d'un train en gare de la Ciotat par les Frères Lumière en 1935 et le film de 1922, The power of love) mais qu'en revanche les enjeux techniques et esthétiques de Avatar (2009) et des films qui ont suivi dans la seconde décennie du $21^{\text {ème }}$ siècle sont spécifiques à cette « nouvelle 3D ».

10. M. Cardon, posté le Mardi 10 avril 2012 sur le blog Slate.fr, en réaction à l'article de J.M. Frodon « Titanic 3D : un ratage, et c'est bien ainsi ».

11. C. Champetier, «Le numérique, à marche forcée ", in Cahiers du cinéma, $n^{\circ} 669$, juillet-août 2011, p. 82.

12. Franz B., « Des nouvelles du front cinématographique (59) : La caverne aux images, deux films sur et dans la grotte Chauvet », 12 septembre 2011.

13. M. Heidegger, "La chose », in Essais et conférences, Gallimard, coll. «tel», 1958, p. 211 : «Rapprocher est l'être de la proximité. La proximité rapproche ce qui est loin, à savoir en tant que lointain. »

14. Comme dans la scène du «corset» de la version en 3D de Titanic qui exacerbe à la fois la texture de la chair et du vêtement tout en intensifiant les effets de profondeur par le jeu des miroirs.

15. G. Deleuze \& F. Guattari, Mille plateaux, Les Editions de Minuit, 1980, p. 616. 
16. À l'inverse d'un très grand nombre d'autres images de ce même film qui usent, voire abusent, de plans jouant des points de fuite de la perspective.

17. J.-M. Frodon, «L'Odyssée de Pi, un petit pas pour la 3D malgré tout », 23/12/2012 (http:// www.slate.fr/story/66385/odyssee-de-pi-un-petit-pas-pour-la-3d-malg : « Le seul apport réel de la technologie et de l'esthétique 3D, la seule vision forte dont seront privés les spectateurs qui ne verront pas le film dans ce format, concerne un motif récurrent et impressionnant: la fusion entre ciel et mer, la translation selon des modalités imprévues, qui troublent les perceptions [...] De diverses manières au cours de la projection, un personnage passe d'un élément à l'autre, ou flotte/vole dans un environnement hybride, qui peut-être le bassin d'une piscine comme l'océan sans limite, une coursive envahie d'eau et les nuages sur Paris, le ciel nocturne ou carrément une vision de l'univers. Tour de prestidigitation visuelle ? Sans doute. Mais ces usages là de la 3D sont, sauf erreur, inédits. Et on sait depuis Méliès qu'avec la mise au point de tels tours, c'est le vocabulaire du cinéma qui s'enrichit, et peut demain exprimer davantage. »

18. G. Deleuze \& F. Guattari, Mille plateaux, op. cit., p. 614-615 : «C'est Aloïs Riegl qui, dans des pages admirables, a donné à ce couple Vision rapprochée-Espace haptique un statut esthétique fondamental. Pourtant, nous devons négliger provisoirement les critères proposés par Riegl (puis par Worringer, et aujourd'hui par Henry Maldiney) pour prendre un peu de risque nous-mêmes, et nous servir librement de ces notions. C'est le Lisse qui nous paraît à la fois l'objet d'une vision rapprochée par excellence et l'élément d'un espace haptique (qui peut-être visuel, auditif autant que tactile). Au contraire, le Strié renverrait à une vision plus lointaine, et à un espace plus optique - même si l'œil à son tour n'est pas le seul organe à avoir cette capacité. »

19. Ibid., p. 596.

20. On trouve parfois des échos manichéens comme dans ce passage qui se réfère au « diabolique » : «Tout ceci pour rappeler que le lisse peut lui-même être tracé et occupé par des puissances d'organisation diaboliques. » (Ibid., p. 600.)

21. Ibid., p. 602: "C'est aujourd'hui, et dans les sens les plus divers, que se poursuit l'affrontement du lisse et du strié ».

22. Ibid., p. 595.

23. Ibid., p. 593.

24. Pour exemple : « Mais rien ne coïncide tout à fait, et aussi tout se mélange, ou passe de l'un à l'autre. C'est que les différences ne sont pas objectives : on peut habiter en strié les déserts, les steppes ou les mers; on peut habiter en lisse même les villes, être un nomade des villes. " (Ibid., p. 601) ou encore «Et pourtant nous retrouvons toujours une nécessité dissymétrique, de passer du lisse au strié, comme du strié au lisse.» (Ibid., p. 606).

25. G. Deleuze, Francis Bacon/Logique de la sensation, op. cit., p. 122-123.

26. G. Deleuze \& F. Guattari, Mille plateaux, op. cit., p. 610.

27. Inanis Aedes, court métrage en 3D réalisé en 2010 par Meradi Omar et Roussel Béranger, étudiants en Master 1 d'Arts et Technologies de l'Image (ATI), Université Paris 8 (Saint-Denis).

28. G. Deleuze \& F. Guattari, Mille plateaux, op. cit., p. 630.

29. Splitscreen : a love story de J.W Griffiths (Film gagnant du Nokia Shorts Competition 2011).

30. G. Deleuze, Francis Bacon/Logique de la sensation, op. cit., p. 151 : « Mais le fait lui-même, ce fait pictural venu de la main, c'est la constitution du troisième œil, un œil haptique, une vision haptique de l'œil, cette nouvelle clarté. C'est comme si la dualité du tactile et de l'optique était dépassée visuellement, vers cette fonction haptique issue du diagramme. »

31. G. Deleuze, Cinéma 2, L'image-temps, Ed. Minuit, coll. «Critique », 1985, p. 22.

32. L'attitude de jeunes enfants face à certaines images 3D du film L'odyssée de Pi notamment.

33. «Réalité augmentée, 3D et tactile : des chercheurs japonais inventent un écran qui permet de “toucher" les images. » (http://www.presse-citron.net)

34. H. Parret, « Spatialiser haptiquement : de Deleuze à Riegl, et de Riegl à Herder », op. cit. 
35. G. Deleuze, Francis Bacon/Logique de la sensation, op. cit., p. 106 : « Nous appelions l'un de ces usages "digital", non pas en référence directe à la main, mais en référence aux unités de base d'un code ". Rappelons que le sens anglais actuel du mot digital (numérique) renvoie à digit qui veut aussi bien dire doigt que chiffre (cf. Harrap's standard French and English dictionary).

36. «Interactive Mirror: Le miroir tactile» (posté le 24 octobre 2008) ; «La société LitStudios vient dévoiler en vidéo son Miroir Interactif tactile » (www.generation-tactile.com/archives-gt/ domotique-tactile/interactive-mirror-le-miroir-tactile-interactif-398)

37. Nous la qualifions ainsi par opposition à la « seconde interactivité » qui valorise, au contraire, l'autonomie (cf. M.H.Tramus, M. Bret, E. Couchot, «La seconde interactivité », in Arte e vida no século XXI, Organizadora Diana Domongues, UNESP; Brasil, 2003.)

38. Pour une étude plus approfondie de cette illusion démiurgique conférée à l'interacteur cf. I. Rieusset- Lemarié, "Entre commande et autonomie : l'esthétique du gestus », in Journée d'étude co-organisée par le séminaire "Action sur l'image" (dirigé par J.L. Weissberg) et le Groupe d'étude « Réseaux » (dirigé par I. Rieusset-Lemarié) le 8 juin 2002 à l'Université Paris 8. (http:// www.hypermedia.univparis8.fr/séminaires/semaction/seminaires/txt01-02/journees0602/ isabel.htm)

39. M. Heidegger, « Les séminaires », in Question III et IV, Gallimard, coll. « tel », 1976, notes, p. 548 : «Il faut partir de l'allemand Zuhandenheit pour voir la symétrie qu'instaure la terminologie de Heidegger entre Zuhandenheit, utilisabilité, et Vorhandenheit, être-là-devant, les deux substantifs abstraits ayant pour radical commun le mot Hand, la main. Mais c'est cependant sur les expressions courantes vorhanden, présent (idée de proximité physique), et zuhanden, sous la main (à portée de main, en main propre, mais aussi : à l'attention de...), qu'ils sont formés et surtout réunis par Heidegger en un couple caractéristique. »

40. Pour une étude plus approfondie de cette idéologie du « à portée de main » dans les médias numériques, $c f$. I. Rieusset-Lemarié, «Les chemins de l'œuvre d'art au-delà de l'utilisabilité (à portée de Net : quelle proximité ?», in Ligeia ${ }^{\circ} 45$, juillet 2003.

41. Pour cristalliser ce pouvoir donateur du geste, Heidegger emploie le terme «Reichen » que François Fédier traduit en français par «porrection »: « Reichen» veut dire la portée d'un geste où quelque chose est procuré. L'ancien français connaissait encore le verbe porriger (tendre, présenter) qui a exactement le sens de reichen. C'est pourquoi on s'est autorisé à traduire das Reichen par : la porrection. (M. Heidegger, "Temps et Être", in Questions IV, tel/Gallimard, traduction française (François Fédier), 1976, note du traducteur, p. 27.) La porrection, c'est un geste qui tend, présente, offre en présent, donne.

42. Pour une étude approfondie des relations entre geste et gestation dans le cadre d'une " culture du soin» cf. I. Rieusset-Lemarié, "Perséphone: la culture du geste ", in Déesses du parfum et de la métamorphose, Berg International Éditeurs, 2011.

43. Pour une approche phénoménologique de l'empathie kinesthésique faisant également appel aux sciences de la cognition cf. la thèse de P. Guisgand (J. Caullier, dir.), « Lire le corps : une voie interprétative » (Université de Lille III, Oct. 2005).

44. Le défaut d'empathie des acteurs virtuels de Final Fantasy a été interprété en relation avec l' «uncanny valley»: «Le plus grand défaut de ce film était l'animation des visages des personnages, qui semblaient sans vie. La cause en est la suivante : découvert dans le monde de la robotique puis dans le monde de l'animation, il existe un effet "the uncanny valley" dont les symptômes sont les suivants: si un objet se rapproche trop de l'allure humaine, alors il va paraître étrange et une sensation d'inconfort se développera chez nous. Ce symptôme est par ailleurs démultiplié lorsqu'il s'agit d'une entité animée. [...] Si un objet ou une entité se rapproche trop de l'apparence humaine, l'empathie du spectateur décroît et une sensation d'inconfort naît. [...] James Cameron était persuadé de pouvoir créer des personnages virtuels grâce à la motion capture faciale notamment et contourner l'uncanny valley ». (H. Saïfi, Incidence 
de la prévisualisation sur la production et l'esthétique des films, Mémoire de fin d'études et de recherche, ENS Louis Lumière, 2011, p. 86).

45. Bien que la "motion capture ", la captation du mouvement du corps des acteurs pour les appliquer ensuite à des personnages virtuels créés par ordinateur, soit utilisée depuis des années, James Cameron et Jon Landau estimaient qu'il manquait un élément majeur au procédé : la lettre «é » devant le mot « motion ». Jon Landau raconte : " Notre idée était de transformer la "motion capture" en "emotion capture". La "performance capture " permet aux acteurs de donner la pleine mesure de leur talent et de leur travail d'interprétation. (cf. « Avatar », in cinemovies.fr)

46. «En permettant de capter les mouvements et expressions du visage (facial motion capture) on a abouti à la technique appelée performance capture : "James Cameron et son équipe ont mis au point un système baptisé performance capture qui surpasse celui de la motion capture”, répond le directeur scientifique du patrimoine de la Cinémathèque française, Laurent Mannoni. » ( 0 . Delcroix, «La révolution Avatar », in Le Figaro, 31/12/2009)

47. Rappelons que Deleuze et Guattari associent l'organique non pas à l'espace lisse haptique mais à l'espace strié : «L'organique, avec sa symétrie, son contour, son dehors et son dedans, se rapporte encore aux coordonnées rectilignes d'un espace strié. » (cf. G. Deleuze \& F. Guattari, Mille plateaux, op. cit., p. 622.)

48. «La Performance capture : le cinéma pour l'éternité ?»: «Mais depuis Avatar et bientôt Tintin, ces capteurs ont presque totalement disparu et laissent place à une petite caméra posée juste devant le visage et qui enregistre le mouvement de tous les muscles du visage, en se référant à des micro-marques. Allégeant les expressions de l'acteur et favorisant donc l'interprétation, cette technique est une vraie bénédiction pour le réalisateur, car c'est [...] une vision plus claire du jeu de l'acteur pendant la "prise », et la précision d'une caméra placée à quelques centimètres du visage! Mais surtout, la principale révolution qu'apporte cette caméra, c'est le tracking des yeux! En effet, c'est probablement la plus grosse difficulté dans la performance capture, avec les mouvements des cheveux et des "extrémités graisseuses ». On l'a remarqué dans Avatar, le regard des Naavi était criant de vie, contrairement à ses prédécesseurs.» (http://www..gameblog.fr/blogs/anfalmyr/p_16630_la-performance-capturele-cinema-pour-l-eternite)

49. Ang Lee cité in T. Baurez, « Il était une fois... L'odyssée de Pi d'Ang lee » : « En ce qui concerne les animaux, j'avais déjà expérimenté les choses avec Hulk, mais il fallait que ça paraisse encore plus réaliste. Le regard d'un tigre ne peut pas tromper celui du spectateur, contrairement à celui d'un géant vert. De plus, la 3D m'obligeait à m'interroger sur le sens de la représentation. Contrairement à la 2D, l'objectif de la caméra ne se substitue plus à l'œil humain. » (http:// www.lexpress.fr/culture/cinema/il-etait-une-fois-l-odyssee-de-pi-d-ang-lee_1200955.html)

50. Pour une analyse de la mise en œuvre du Cinéma 3D au service d'un effet de distanciation, en particulier dans le film de M. Scorsese, Hugo Cabret, $c f$. I. Rieusset - Lemarié, «Les corps au cinéma à l'épreuve de la 3D : immersion, distanciation et empathie ", dans Figures de l'art, $\mathrm{n}^{\circ} 25$, « Les nouveaux dispositifs immersifs », septembre 2013.

51. Pour l'analyse de l'empathie en termes de résonance $c f$. E. Couchot, Des images, du temps et des machines dans l'art et la communication, Editions Jaqueline Chambon-Actes Sud, 2007.

52. Pour une analyse plus approfondie de cette notion de "gestus " $f$. I. Rieusset-Lemarié, «Nouvelles images et cinéma: de l'interaction des codes esthétiques au devenir corps des images-temps », in Médiamorphoses n², INA, juillet 2001.

53. G. Deleuze, L'image-temps, op. cit., p. 250 : «Ce que nous appelons gestus en général, c'est le lien ou le nœud des attitudes entre elles, leur coordination les unes avec les autres, mais en tant qu'elle ne dépend pas d'une histoire préalable, d'une intrigue préexistante ou d'une imageaction. Au contraire, le gestus est le développement des attitudes elles-mêmes et, à ce titre, opère une théâtralisation directe des corps [...]. C'est la grandeur de l'œuvre de Cassavetes, avoir défait l'histoire, l'intrigue ou l'action, mais même l'espace, pour atteindre aux attitudes comme 
catégories qui mettent le temps dans le corps, autant que la pensée dans la vie. Quand Cassavetes dit que les personnages ne doivent pas venir de l'histoire ou de l'intrigue, mais l'histoire, être sécrétée par les personnages, il résume l'exigence d'un cinéma des corps [...]. C'est l'enchaînement formel des attitudes qui remplace l'association des images. »

54. Le court métrage Je m'appelle NATHAN de Benoît Berthe (2012) donne accès à l'intériorité de la tête du personnage virtuel, en transformant le maillage de sa maquette de construction en une cage au sein de laquelle un oiseau réclamant ses graines à tue-tête trouble sa "paix intérieure " qu'il ne pourra retrouver que lorsqu'il se sera accordé au mouvement de cette "altérité » interne. Cette figuration métaphorique prête à ce personnage une forme sinon "d'inconscient ", à tout le moins d'intériorité, qui favorise l'empathie du spectateur.

\section{RÉSUMÉS}

Si certaines œuvres du cinéma 3D vérifient le rôle privilégié de la «main de l'œil» dans l'haptique deleuzien d'autres, par leur irréductibilité à l'opposition entre espace haptique lisse et espace optique strié, nous conduisent à dépasser cette axiologie dualiste, non sans interroger la réversibilité même du tactile qui se manifeste par la rationalisation de l' "œil digital » et l'illusion de maîtrise de la main démiurgique. Irréductible à ces écueils, la «profondeur haptique " tend non seulement à dépasser ce système duel mais à développer la potentialité empathique du toucher, que ce soit sous la forme de l'empathie kinesthésique développée à l'égard des acteurs virtuels ou par la mise en jeu d'un « regard haptique » ouvert au vertige de leur altérité radicale.

If some 3D movies seem to testify the privileged role of "the eye's hand" in Deleuze's theory of haptic, other ones, through their irreducibility to the opposition between the smooth haptic space and the striate optical space, lead us beyond this dualistic axiology not without questioning the reversibility of the tactile which appears in the rationalization of the "digital eye" and the delusion of control by the demiurgic hand. The haptic "depth" tends not only to bypass this dual system but to develop the empathic potentiality of the sense of touch, either through the kinaesthetic empathy developed towards virtual actors or through the involvement of a "haptic outlook" open to the dizziness of their radical otherness.

\section{AUTEUR}

\section{ISABELLE RIEUSSET-LEMARIÉ}

Maître de conférences HDR à l'Université Paris 1 Panthéon - Sorbonne. Elle appartient à l'Institut de recherches ACTE (UMR). Elle est membre du comité exécutif de l'ESA (European Society for Aesthetics). Dans son ouvrage La Société des clones à l'ère de la reproduction multimédia (Editions Actes Sud, 1999), elle a analysé les enjeux des « humains virtuels » et des créatures artificielles à la lumière des perspectives ouvertes par Walter Benjamin. Ces dernières années, elle a poursuivi ses recherches dans le cadre de nombreux travaux et publications portant sur les acteurs virtuels et sur les dispositifs d'immersion dans le Cinéma 3D, sur les fondements de l'esthétique dans l' aisthesis et tout particulièrement sur la revalorisation des sens discrédités de l'odorat, sur les 
relations entre images et gestes (le rôle du « gestus » et du « cinéma des corps » chez Deleuze) et sur la fonction privilégiée du geste dans les esthétiques intermédias qui s'inscrivent plus

largement dans son champ de recherche axé sur les relations interartiales mettant en jeu la synesthésie. 